CITE AS:

Konrath, S. (2014, in press) Positive technology. Using mobile phones for psychosocial interventions. In Encyclopedia of Mobile Phone

Behavior, Zheng Yan, IGI Global.

\title{
Positive technology: Using mobile phones for psychosocial interventions
}

\section{Sara Konrath}

Research Center for Group Dynamics, Institute for Social Research, University of Michigan Psychiatry Department, University of Rochester Medical Center

United States

\section{Acknowledgements}

Sara Konrath was supported by grants from The John Templeton Foundation, via the Psychology of Character (Wake Forest University), and directly from the sponsor (Grant \# 47993). 


\begin{abstract}
This chapter reviews the use of mobile phones in psychosocial interventions. Specifically, it reviews research studies that have used text messages (SMS) or smartphone applications (apps) to improve people's mental health, psychological well-being, or social relationships. Psychosocial interventions are emerging from the larger and more established mobile health (mHealth) literature of physical health interventions. The scientific knowledge of psychosocial interventions is currently quite limited, with only a few published large randomized control trials. Most of those are limited to North American or European participant samples. The advantages and disadvantages of mobile interventions are discussed, along with recommendations for best practices. The success of future research is dependent upon more researcher-friendly tools to implement interventions.
\end{abstract}




\section{Intellectual history}

\section{INTRODUCTION AND OVERVIEW}

Mobile phones are ubiquitous: $91 \%$ of Americans own one (Center, 2012), and 61\% of those are smartphones (Nielsen, 2013b). Smartphones are extremely versatile, functioning like personal computers, and are an increasingly important part of people's daily lives. In one study, smartphones were within arm's reach $50 \%$ of the time, and in the same room $90 \%$ of the time (Dey et al., 2011). Researchers and clinicians in a variety of fields, including medicine and public health, have taken note of mobile phones as potentially powerful interventions.

Mobile phone interventions, including text messages and smartphone applications, are part of the broader use of electronic tools to improve physical health (also called e-health or mHealth). mHealth is defined as "the use of mobile and wireless technologies to support the achievement of health objectives" (Kay, Santos, \& Takane, 2011). These started (and continue) via web-based platforms, and evolved with the rise of widespread mobile phone usage. The first known studies to examine the use of text messaging (or SMS - short message service) were published in 2003, making this a relatively young research discipline. Victoria Franklin of the University of Dundee, UK (Franklin, Waller, Pagliari, \& Greene, 2003) and Stephanie Bauer of Heidelberg University, Germany, (Bauer, Percevic, Okon, Meermann, \& Kordy, 2003), are among the earliest published authors in the field.

Since these early studies, there have been a large number of intervention studies to improve physical health outcomes, and currently there are a number of published review articles and meta-analyses (Cole-Lewis \& Kershaw, 2010; Fiordelli, Diviani, \& Schulz, 2013; Fjeldsoe, Marshall, \& Miller, 2009; Herbert, Owen, Pascarella, \& Streisand, 2013; Krishna, Boren, \& Balas, 2009; Liang et al., 2011; Militello, Kelly, \& Melnyk, 2012; Park, Howie-Esquivel, \& Dracup, 2014; Shaw \& Bosworth, 2012; Whittaker et al., 2009). Since text messages are inexpensive, comparatively simple to program, and available to any mobile phone owner, the majority of physical health mobile-based studies have used text messages to deliver interventions, rather than smartphone applications (see Table 1). One recent review concluded that "the potential of smartphones does not seem to have been fully exploited yet" (Fiordelli et al., 2013, p. 7). To date, the vast majority of these studies have taken place in Europe and North America. Studies have only rarely been conducted in developing countries, where the need for such tools is high because of relatively low access to physical health resources. Interventions have ranged from relatively short durations ( 2 weeks) to relatively long-term (14 months), with the frequency of the interventions ranging from 1 time per month to 6 times per day. The majority of studies (between $60 \%$ to $100 \%$ ) have found positive health change on the outcome measure of interest, although this might be in part due to publication bias. The reviews demonstrate that follow up assessments are rare, making it unclear how long these effects last.

\section{$<$ INSERT TABLE 1 HERE $>$}

\section{Current scientific knowledge}

Building on the powerful potential that mobile technology has for reaching people in vivo, social scientists are developing innovative technology-based approaches to help individuals initiate and sustain behavior change in psychological and social ("psychosocial") domains, which include mental health, well-being, and social relationships. Although there has been much research on physical health behaviors and outcomes, the use of mobile phone technology to alter psychosocial outcomes is much more recent and much less common. One recent review found that psychosocial measures comprised only $9 \%$ of outcomes (Fiordelli, et al., 2013). As will become clear in this chapter, to date the literature on so-called "positive technologies" is only emerging (Riva, Banos, Botella, Wiederhold, \& Gaggioli, 2012), and the quality of research varies dramatically - with well-designed pre-post randomized control trials relatively rare. It is 
difficult to identify leading scholars in this area because it is so new and because scholars are making contributions across many different disciplines. However, leading contenders would be David Mohr and Stephen Schueller at the Center for Behavioral Intervention Technologies at Northwestern University, and Andrea Gaggioli and Giuseppe Riva at the Catholic University of Milan, Italy.

\section{Advantage and disadvantages of mobile interventions}

As with any research tool, there are advantages and disadvantages of using mobile phone technologies as psychosocial interventions, compared to traditional in person approaches.

Advantages. One advantage to mobile phone interventions is that they can broaden the demographic groups that can be reached. For example, many psychology studies rely on college students samples for convenience, which limits the generalizability of conclusions made. Mobile interventions allow researchers to study more diverse groups in terms of age, socioeconomic background, and race / ethnicity. Smartphone penetration is higher among African-Americans (33\%) and Hispanics (45\%) than Caucasians (27\%; (Nielsen, 2013a). Smartphone use may be highest among low-income groups due in part to the relative affordability of month-to-month 3G/4G contracts when compared to home-based WiFi (Klasnja, 2013). Mobile phones can help to bridge the "digital divide" (Chang et al., 2013; Ginossar \& Nelson, 2010). In addition, mobile interventions can target specific groups such as people who are living with or at risk for mental illnesses, people entering specific life stages (e.g. expectant parents), and people in remote locations, including those in developing nations.

Mobile phones are relatively low in cost, convenient and easy to use, and portable. Their wide use makes it possible to collect data from very large samples and disseminate interventions very broadly. Their near constant access means that researchers can deliver interventions or collect data via experience sampling multiple times, without researchers and participants directly interacting. One of the difficulties with implementing behavior change is that people have habitual ways of thinking, feeling, and interacting with others, among other behaviors. With respect to health conditions, people also find it difficult to adhere to behaviors that maximize their health, such as checking their glucose levels or taking medications. Mobile interventions can help to build mentally and physically healthy habits because they can be repeated across time, and specifically targeted to high-risk time periods (e.g. after a meal for a smoker). They can also be individually tailored based on characteristics of participants (e.g. name, gender, age, etc). Moreover, the fact that mobile interventions are applied within everyday contexts may help people to generalize these behaviors across a variety of real-life settings (e.g. work, school, home, leisure).

Mobile-based technologies offer a number of advantages for interventions specifically focused on mental health (Donker et al., 2013). For example, they have potential to improve the accessibility of treatment and increase adherence to medication (Granholm, Ben-Zeev, Link, Bradshaw, \& Holden, 2012). There is also the potential for researchers or clinicians to track the progress of treatment via real-time data collection. Moreover, there is potential for personalization, which may increase motivation to continue treatment programs. Finally, the fact that treatment can occur across a variety of settings may help treatments to generalize more widely than the typical 1 hour therapy session. However, to date there are only a few known published studies using SMS or smartphone apps to help improve the symptoms of people with mental illnesses (see Table 2). Thus, the potential for mobile-based interventions in mental health has been underutilized.

There are some advantages that are specific to SMS-based interventions. Nearly all phones have text messaging capability and it is widely used. Users do not need to be too technologically savvy to learn how to text message. Text messages are asynchronous, so that users can access text messages at times that are convenient to them. If phones are turned off, text messages are delivered when they are turned back on. For researchers, any type of mobile-based intervention is currently difficult to implement, but it is easier and less costly to develop, test, and 
implement a text-message based study than to develop, test, and implement a smartphone app intervention study. People with basic programming skills can work with a text message provider to administer their text messages, whereas most academics do not have the technological expertise to program a high-quality app by themselves.

There are also some advantages that are specific to smartphone-based interventions. Most interventions to date are relatively straightforward, typically adapting something that worked well in face-to-face or textual formats into mobile-based interventions. Yet smartphones have a massive potential for both intervention delivery and data collection that go beyond simple didactic-based interventions (Miller, 2012). For example, interventions can be interactive (e.g. including 2-way communication between participants/patients and researchers/clinicians), gamelike, and involve adaptive learning (e.g. learning participants' moods and providing feedback). Smartphones can also collect a wide variety of objective data such as physiological variables (e.g. heart rate, movements via in-phone accelerometers), location data (via in-phone GPS systems), auditory information (e.g. words being spoken), visual information (e.g. pictures of participants' food), and reaction-time data, among others (Miller, 2012). With increasing technological sophistication of these phones, in the future it might be possible to target interventions to the highest risk times based on deviations from participants' own baseline data (e.g. mood: for an early prototype, see (Burns et al., 2011).

Disadvantages. Mobile interventions also have a number of disadvantages. From the researcher's perspective, such studies are labor intensive and expensive, because of the limited user-friendly applications available to help to design and implement such studies (Miller, 2012). Most researchers have no idea how to program, debug, and pilot test mobile-based interventions. In the early days of internet research, there were similar problems. Yet the development of easy to use survey creation portals (e.g. Survey Monkey, Qualtrics) opened the doors for any researcher to conduct high quality online studies. There is a promising new program, SurveySignal (Hofmann \& Patel, 2014), that allows researchers to easily send text-message surveys as to smartphone owners (see Table 3).

Even when such tools become more widely available, the additional challenge will be to analyze the massive amounts of data that mobile intervention studies can collect (e.g. experience sampling studies via smartphones; Miller, 2012). Many scholars simply decide to focus on the most basic outcomes, but this greatly limits the amount that we can learn from using these tools. Another point of consideration is that although mobile phones (and especially smartphones) have been widely adopted by a wide variety of people, it may still be challenging to recruit certain populations. For example, the elderly, children, and transient populations may be difficult to recruit for mobile intervention studies (Miller, 2012). Thus, researchers must consider whether delivering interventions via mobile phones makes sense for their participant group of interest.

One other disadvantage of relying on technological interventions is that behavior intervention technologies can quickly become obsolete. Thus, researchers need better and faster evaluation and dissemination methods (Schueller, Muñoz, \& Mohr, 2013). In addition, there are often technical problems that come up while implementing mobile-based interventions, so researchers must have a technical support plan (e.g. who do participants contact if they miss a text message?). Mobile intervention studies also share some disadvantages as internet studies.

Although there is an increase in ecological validity in mobile-based interventions, this may come at the expense of having low control over participants' daily environments. For example, participants may complete intervention materials while they are distracted, socializing, or otherwise occupied. This means that effect sizes are likely relatively smaller compared to those in more controlled lab-based interventions, and thus, larger samples are needed in order to detect an intervention's effect.

There are also some disadvantages from the user's perspective. First, in order to participate in such interventions, people must have access to a phone and be literate. Although it is true that cell phones and usage plans cost money, the cost has been declining over time. Some 
researchers add the cost of phones into their study budgets, and provide participants with a phone for the duration of the study, at the risk of it being lost, stolen, or broken. The possibility of phones being lost or stolen-regardless of whether they are owned by the participant or borrowed-is a threat to intervention efficacy. It is also possible to conduct research on illiterate populations by designing voice-based or image-based interventions. However, such interventions are extremely rare.

There are also ethical issues to consider when conducting mobile-based intervention studies. For example, very intimate data can be collected with smartphones, so researchers must be careful to protect participants' privacy and confidentiality. Related to this, when collecting data from participants' environments (e.g. images, voices), other people who have not consented to the study may be unobtrusively recorded. This raises issues of informed consent in addition to privacy (Miller, 2012).

For apps specifically, users must have a smartphone, and sometimes apps must be downloaded, which puts a burden on participants. Also, apps typically only work in a single operating system unless researchers program them across multiple platforms. For SMS specifically, text messages can cost participants who do not have unlimited plans. In addition, it is easy to implement interventions using text messages, but more difficult to collect data from participants this way.

There are a number of disadvantages to consider with mental health interventions specifically (Donker, et al., 2013). For example, technical problems over the course of a mobilebased treatment study may have serious consequences, especially in the case of serious mental illnesses. Related to this, researchers must have a clear plan of how to respond to crises that are identified during tracking. With mental health patients, privacy concerns and the security of data are especially important. Moreover, it is possible that patients may feel isolated or disconnected if mobile interactions replace face-to-face therapeutic interactions (Berry \& Lai, 2014). Finally, people often share their phones, and in the case of mental health interventions, privacy and intervention efficacy concerns would be magnified in these cases.

\section{Mental health interventions}

\section{REVIEW OF PSYCHOSOCIAL INTERVENTIONS}

Mobile technology has a huge potential for providing mental health resources and support. Many people cannot access mental health services because of income, insurance status, time availability, or limited providers available. In addition, mental health conditions are strongly stigmatized, making mobile-based services especially appealing. A recent systematic review of smartphone apps for improving mental health outcomes (e.g. depression, anxiety, substance use) found only 8 studies total that used randomized control experimental designs or pre-post designs, from a total of 5464 initial abstracts (Donker, et al., 2013). All except one of these studies were feasibility or pilot studies, and only one study included a follow up beyond the post-test intervention period. Because of this, it is unclear whether mobile interventions can have longterm, sustainable, effects. With thousands of mental health apps available to download from app marketplaces, it is remarkable that only 8 at the time of the systematic review paper were evidence-based.

Depression. The most common mental health mobile intervention targets depression. One app, mobiletype, tracks users' experience to help increase emotional self-awareness, in the hope of reducing depressive symptoms (Kauer et al., 2012). Emotional self-awareness involves recognizing and identifying emotions, the situations that trigger them, communication about emotions, and developing coping strategies. Mobiletype teaches emotional self-awareness by having users regularly report on their mood, recent stressful events, responses to those events, alcohol use, and drug use. Users also report their current activities, location, companions, and sleep, exercise, and eating habits. In a randomized control trial, 114 participants aged 14 to 24 
with depression were randomly assigned to use a control version of the app (without the emotional experience or substance use questions) versus a full version of the app that included all its components. Participants recorded at least 2 entries per day for a period of two to four weeks. They were then assessed immediately after using the app, and 6 weeks after that. Participants in the treatment group had higher emotional self-awareness, and in turn, this was linked to fewer depressive symptoms. There were no differences in rumination across the two conditions.

Another app, Mobilyze!, passively records contextual features such as users' location, ambient light, accelerometer data, and recent calls, and attempts to match these features with selfreported user information (e.g. mood, recent social interactions) to learn users' patterns and determine the need for help (Burns, et al., 2011). A pre-post study examined the efficacy of this app in 8 participants with major depression disorder for an 8 week period, in combination with online behavioral training and email/phone support from clinicians. The study found that participants had fewer symptoms of depression and anxiety by the end of the study. However, since there was no control group, it is impossible to know if this would have occurred without the app. Also, the small sample size makes it difficult to generalize more broadly. Finally, because the treatment included two other components (online behavioral training and clinician support) besides the app, the specific effect of the app itself is to be determined.

Mobile interventions have been strongly influenced by online interventions, yet almost no research exists comparing the efficacy of the same intervention across each delivery mode. One important study did just this by examining whether a cognitive behavior therapy program would be more effective for 35 depressed participants when delivered over the phone versus on the internet (Watts et al., 2013). Participants received 6 lessons over a period of 8 weeks, and completed brief homework assignments in between each lesson. The lessons consisted of a comic book-like story about a person with depression. The first two lessons came with clinician support (via phone or email), after which clinicians were available upon participants' request or if they noticed that participants' scores indicated increasing levels of distress. The study found that there were no condition differences in the percentage of participants who completed all of the lessons (69\%), and that participants in both groups experienced similar declines in depressive symptoms. However, there was no control group, so it is unclear if the treatment itself was the cause of these declines. In addition, the sample size was small, which made it difficult to detect differences between the two groups if they did occur. It is also impossible to disentangle the effect of the lessons versus the clinician support.

One final study on depression used an SMS-based intervention (Aguilera \& Muñoz, 2011). Participants were 12 low income patients enrolled in group cognitive behavior therapy. Using a pre-post design, participants received 2 text messages per day that asked about their mood and also had a brief therapy-based homework question (e.g. How many positive social interactions did you have today?). Most participants reported that they enjoyed the text message addition to their group therapy. Although the results found a non-significant $(\mathrm{p}=.15)$ decline in depressive symptoms over the 2 to 4 month study period, it is unclear whether this marginal decline was due to the group therapy or the text messages. A larger sample size and a control group are needed in future research.

Anxiety. Anxious people have an exaggerated response to threatening stimuli, or high threat bias (Bar-Haim, Lamy, Pergamin, Bakermans-Kranenburg, \& van IJzendoorn, 2007).Attention-bias-modification training (ABMT) directs people away from threatening stimuli and toward more non-threatening stimuli. ABMT uses the dot-probe task, in which neutral and angry facial expressions are presented together, then participants are asked to make fast judgments about which way an arrow is pointing. Typically, faster responses to a cue in an angry location indicate more threat bias. However, people can be trained to focus more on nonthreatening stimuli if the arrow (or other probe) is consistently paired with neutral stimuli only (MacLeod, 1995). This teaches them to direct their attention to neutral stimuli in the face of 
threats. Indeed, ABMT has been shown to reduce threat bias, and as a result, reduce anxiety (Beard, Sawyer, \& Hofmann, 2012; Hakamata et al., 2010; Hallion \& Ruscio, 2011).

There are two well-designed studies that test the efficacy of administering ABMT via smartphone apps. In one study, 76 highly anxious college students ( $+1 \mathrm{SD}$ above the mean) were randomly assigned to receive a gamified ABMT app versus a placebo control app (Dennis \& O'Toole, 2014). In addition, half of participants engaged with the app for 25 minutes while the other half engaged with it for 45 minutes. The app adapted the classic ABMT dot-probe task with animated characters and game like instructions. In the treatment app, 100\% of trials tried to shift players' focus to the neutral face rather than the angry face, while the control app was the same game, but with a $50 \%$ focus on neutral versus angry faces. Participants in the treatment conditions had lower self-reported and observed anxiety and stress reactivity during a stressful situation in the laboratory (e.g. giving a speech), regardless of the duration of treatment ( 25 versus 45 minutes). However, only participants in the longer treatment group had less threat bias.

Another app used the classic ABMT dot-probe task with pictures of human faces showing disgust or neutral responses (Enock, Hofmann, \& McNally, 2014). Participants were 251 non-clinical adults who were randomly assigned to use the treatment versus control app for a period of 4 weeks, or to a waitlist control condition. In the treatment group, $100 \%$ of trials shifted participants' focus to the neutral face rather than the disgust face, and in the control group, the ratio was $50 \%$ disgust versus neutral focus. Overall, there were declines in social anxiety, general anxiety, and depressive symptoms, regardless of which app (treatment versus control) participants used. However, there was no change in the waitlist control group. In addition, both the treatment and control app led to declines in threat bias over time, but there were significantly greater declines in treatment group. The researchers concluded by suggesting that perhaps any mobile treatment app would help to reduce symptoms anxiety and depression. Future research is needed to better understand which ingredients are most likely to help reduce anxiety symptoms.

Schizophrenia. In an SMS-based intervention study, participants were 62 schizophrenic patients (Pijnenborg et al., 2010). All participants first completed 6 weekly psychoeducation sessions, then received a set of tailored goals to achieve during the 7 week treatment period (e.g. taking medication, mental health appointments). Using a quasi-random experimental design, the first 33 participants were assigned to an active treatment condition (baseline, intervention, follow up) and the next 29 were assigned to a wait-list control condition (baseline, waiting period, then intervention and follow up). While receiving the text messages, the percentage of the goals that participants achieved increased. However, this decreased when participants stopped receiving text messages in the follow up period. So, the tailored text message intervention had some success at improving treatment outcomes, but in such clinically severe populations, treatment might need to be ongoing to be successful.

Another SMS-based intervention targeted three treatment domains: medication adherence, socialization, and auditory hallucinations (Granholm, et al., 2012). Using a pre-post design, 55 schizophrenic patients received 3 sets of interactive texts corresponding to these three domains, 6 days a week, for a 12 week period. Personalized information was included in the text messages, for example, their own views about why medications were helpful were presented for the medication adherence domain. Thirteen participants dropped out, and these tended to have more severe negative symptoms and a lower IQ. Of those who completed the study, the response rate was high (between $83 \%$ and $86 \%$ depending on the domain), and $86 \%$ of phones were returned intact. Each day of receiving the treatment predicted an increase in remembering to take their medication, an increase in socialization behaviors, and a lower likelihood of auditory hallucinations. These results were promising, but future research would need to include a control group in order to determine whether the intervention actually caused these changes.

In an app-based intervention study, participants were 33 schizophrenic patients (BenZeev et al., 2014). Using a pre-post design, the study examined the efficacy of an app called FOCUS over a one month period. FOCUS targets a number of domains, including psychosis 
symptoms, social functioning, mood, sleep, and medication adherence. At baseline participants selected two of these domains to target during the treatment phase. All participants also focused on medication adherence. Participants then completed three daily assessment modules measuring their mood and recent symptoms. The modules were interactive, with responses tailored to their baseline responses. All content was also available at any time for self-management of symptoms and coping ideas. Overall, participants used the app on $87 \%$ of the intervention days, and about 5 times per day that they used it. Lower functioning participants were just as likely to use the app as higher functioning ones. After one month of using FOCUS, participants had fewer symptoms of schizophrenia and depression. However, there were no changes in their insomnia scores or their beliefs about the use of medication (which were generally positive to begin with). Moreover, the more participants used the app, the fewer symptoms of depression that they had, however, there was no relationship between usage and schizophrenia symptoms. FOCUS appears to be a promising intervention, however, with no control group, a low sample size, and a relatively shortterm implementation, it is difficult to make confident conclusions about the efficacy of it. Future research should conduct larger and longer randomized control trials.

Summary. There are nine mental health interventions currently available: 4 on depression, 2 on anxiety, and 3 on schizophrenia. The majority of them (6) are apps and the rest (3) are SMS-based. Only 4 of the studies are randomized control trials, which means that for the majority of the currently available interventions, it is unclear whether the mobile-based intervention is the actual cause of the reported outcome (Campbell \& Stanley, 1963). More research is needed using high quality randomized control trials, with large sample sizes, and among other clinical populations.

\section{Psychological well-being interventions}

Mobile phones have just begun to be used as "positive technologies" (Riva, et al., 2012) to promote happiness, meaning, and social connectedness. As such there is only limited evidence on whether this "applification of mental wellbeing" (Gaggioli \& Riva, 2013) can indeed help to meet our deepest human needs. In this section, I discuss interventions designed to help nonclinical populations achieve higher well-being, broadly defined to include a variety of intrapersonal outcomes (See Table 2). To date, all known interventions are based on smartphone apps: I know of no empirically evaluated SMS based intervention.

It may be possible to use technology to increase happiness. The Live Happy app allows users to practice 8 different happiness building activities (e.g. savoring the moment, gratitude journal, remembering happy times; (Parks, Della Porta, Pierce, Zilca, \& Lyubomirsky, 2013). Participants were 327 naturalistic users of the app who reported their happiness on at least 2 occasions between 3 to 14 days apart. Thus, the study used a naturalistic pre-post design. The researchers found that participants had increased happiness and overall positive mood over time, and that more app usage and more varied happiness activities were associated with greater increases in happiness. This represents some promising pilot data about the potential efficacy of

the Live Happy app. However, future research would need to include a control condition. Without comparing changes in happiness in a control group, it is impossible to make definitive conclusions about the efficacy of the app.

Smartphone apps could also be used to promote gratitude. For example, one recently developed app aims to teach people to be more aware of good things in their life by sending them daily reminders to be grateful (Runyan et al., 2014). In a randomized control study, participants who received four gratitude reminders throughout the day for 14 days had more positive and stable moods compared to control group participants who either received only one gratitude reminder per day, or control instructions (e.g. "List 1 or 2 of the major things you have been doing in the last hour"). However, in a one month follow up test, the gratitude app had no longterm effects on gratitude, mood, mental health indicators (depression, anxiety, stress), or satisfaction with life. Thus, future studies should vary the dosage to examine whether there is a point at which gratitude training would lead to long term results. 
One other way to promote well-being might be increase people's awareness of their emotions and moods, and how these feelings affect their behaviors and relationships. Indeed, a recent study examined the effect of regular self-reporting of moods (Morris et al., 2010). Using a pre-post design, 10 participants who indicated high stress but were otherwise mentally healthy used an app called Mood Map to rate the valence and arousal of their mood every morning, evening, and several times during the day (every 30 minutes to 3 hours, based on their desired frequency), for a 1 month period. After recording their moods, participants could click on "mobile therapies," which were short exercises (less than 1 minute each) based on cognitive behavior therapy (e.g. breathing techniques, physical relaxation exercises). Participants completed weekly interviews with the research team while using the app. The results were not reported in aggregate, however, five case studies were presented, with participants showing improvement in handling stress and greater comfort with their emotions. This study is promising, but has a number of limitations including a very small sample size and no control group. In addition, it is unclear which part of the app was most beneficial: the mood mapping or the short therapies. Thus, future research should disentangle these two aspects.

Another recent paper had the dual purpose of introducing a flexible app that researchers could use for data collection (iHabit - see Table 3), and testing whether increased self-awareness affected students' wise use of time. In the study, 81 students were randomly assigned to receive self-awareness reminders 5 to 7 times per day during three weeks of the semester, and the control group completed pre- and post-test measures only (Runyan et al., 2013). The self-awareness reminders asked students what they were doing in the last 20 minutes (e.g. academics, exercising, using electronics recreationally). Participants who received self-awareness reminders reported that they were indeed more aware of how they spent their time, and they also reported a slight increase in studying time compared to control participants. Although this study did not directly measure well-being, it is possible that being more self-aware could increase it. Future research should test this possibility.

There are numerous mindfulness apps available, and one recent paper reviewed a large number of them, finding that so far, no randomized trials exist examining the effect of using such apps on well-being (Plaza, Demarzo, Herrera-Mercadal, \& García-Campayo, 2013). However, one more recently published paper provides some preliminary data. The AEON app aims to help users to increase their mindfulness using a thought distancing approach (Chittaro \& Vianello, 2014). Via an interactive screen, users write down worrisome thoughts and then play with virtual waves to make the thoughts fade and disappear, as if they are being gently erased by the water. Using a within-subjects experimental design, 32 naïve meditators were randomly assigned to play with the AEON app or to participate in two control group mindfulness exercises (presented in counterbalanced order). The first asked them to imagine their worrisome thoughts on clouds that were floating away, and the second asked them to write their worrisome thoughts on index cards and throw them in the trash. The study found that the AEON app helped users to separate themselves from their negative thoughts. Users also enjoyed the app and found it easier to use than the other mindfulness methods. Based on prior research finding that mindfulness seems to positively influence well-being (Brown, Ryan, \& Creswell, 2007), it is possible that the app could have beneficial effects. However, this study did not directly examine the app's effect on wellbeing, and more research is needed.

Two studies from the same lab examined the efficacy of implementing relaxation training exercises in high stress groups via a smartphone app (Villani et al., 2012; Villani et al., 2013). In two small pilot studies $(\mathrm{N}=30 ; \mathrm{N}=16)$, the researchers randomly assigned female oncology nurses to either receive an app with relaxation training videos or one with neutral videos. All participants were then exposed to a stressful video of a hospital room with a patient with cancer. Across the two studies, the researchers found that the app caused a significant decline in state levels of anxiety, and an increase in coping skills, over a 4 week period. These results are promising but 
future research should replicate these results in larger samples and among more general populations.

Self-affirmation is the tendency to live in alignment with one's personal values (Sherman \& Cohen, 2006; Steele, 1988), and one recent study tested the efficacy of a self-affirmation app called Viary (Ly, Dahl, Carlbring, \& Andersson, 2012). Via a pre-post design, 11 mentally healthy participants completed a 45 minute psychoeducation module online about living in tune with one's values, and then used Viary for four weeks. The app helps users to determine their own personal values and then gives them guidelines about how to break them down into concrete, achievable steps. Although at the mean level there were slight rises in life satisfaction, and declines in symptoms of depression and anxiety after using the app, these changes were not statistically significant. However, participants' increased in their perception that they were living in accordance with their values, and also increased in psychological flexibility. Future research should recruit a larger sample size and a control group. Moreover, it is unclear if changes are because of the app itself, or the app in combination with the psychoeducation module. Thus, future research also needs to disentangle these two treatments.

Summary. Of the seven psychological well-being apps, only four of them used randomized control trials. The other three use a pre-post design, which makes it impossible to know how much of the change found is attributable to the app itself versus other factors (e.g. maturation, history, statistical regression; (Campbell \& Stanley, 1963). Taken together, there needs to be more research in order to determine whether mobile phones can help to increase psychological well-being.

\section{Interventions for social relationships}

Social connectedness. Mobile media have proven to be a double-sided coin in terms of social relationships. On the one hand, mobile media can threaten people's social connections. Many screen-filled moments happen at the expense of real-live social interactions. Several scholars have suggested that new media, including mobile technologies, may impair relationship bonding processes (Konrath, 2012; Turkle, 2012). For example, one well-designed experiment found that the mere presence of a cell phone in the room resulted in lower feelings of closeness and trust between two conversing strangers (Przybylski \& Weinstein, 2013). Studies have also found that simple reminders of cell phones can reduce empathy and make people less likely to help others in need (A. Abraham, Pocheptsova, \& Ferraro, 2012; Przybylski \& Weinstein, 2013). It may be less surprising then that our research has found that declines in college students' empathy coincided with the rise of social media and mobile phone usage (Konrath, O'Brien, \& Hsing, 2011).

Yet, mobile phones can also make people feel more connected to others ("Always connected: How Smartphones and Social Keep Us Engaged," 2013; Coyne, Stockdale, Busby, Iverson, \& Grant, 2011; Faulkner \& Culwin, 2005; Padilla - Walker, Coyne, \& Fraser, 2012; Pettigrew, 2009). They can help people to feel closer to their loved ones even when there is physical distance, which is likely one of the reasons that they are so attractive and widely used.

Most of the prior research on mobile phones and social connectedness has correlated natural usage patterns with social connectedness variables. However, there are two known studies that use mobile phones as psychosocial intervention tools to build stronger social bonds. In one study, there was no intervention per say, but instead, 49 students in technical communication classes were randomly assigned to use SMS only in their class group projects versus non-SMS communication modes (Lam, 2013). The researcher found that students who were only allowed to use SMS had more frequent communication with their team members, and felt more socially connected to them. However, there were no differences between the two conditions in their attitudes toward the group overall.

Another study randomly assigned 90 students to receive text messages designed to increase empathy, versus two control groups: control messages designed to increase self-esteem, 
and a no-treatment control group (Konrath et al., 2014). Using a pre-post experimental design, participants received 6 daily messages for a 14 day period, and then were assessed on a number of empathy-related variables. Participants who received the empathy-building text messages donated more time to someone in need and also had more prosocial motivations for helping. Yet, in selfreport measures, they saw themselves as having less empathic traits than control group participants. This is perhaps because the heavy dosage of messages reminded them of how they deviated from the highest standard of kindness. A larger scale randomized control trial is in progress.

Aggression. Again mobile technology can be a double-sided coin when it comes to bullying and aggression. Much research finds that new technologies, including mobile phones, can be use to ostracize, humiliate, and bully others, often anonymously and persistently (Kowalski, Giumetti, Schroeder, \& Lattanner, 2014). Yet, a text-message based bullyingprevention program has also recently been developed (DoSomething.org, 2014). The Bully Text is a scenario-based text message program in which users experience what it might be like on a first day of school. They are given messages describing a social scenario, for example: "At your locker you see people looking at their phones and whispering. You notice you have a text. Curious? OPEN IT or IGNORE IT." Users then choose a response, and the scenario continues based on their response. The scenarios involve either being a bully, bystander, or victim of bullying, and theoretically this program may help teens to practice responding to such situations. However, to date there is no empirical evidence on its efficacy at reducing bullying attitudes or behaviors.

Only two known studies have examined the efficacy of using text messages to reduce aggressive traits and behaviors (Konrath, et al., 2014; Rajabi, Ghasemzadeh, Ashrafpouri, \& Saadat, 2012). In the same study that found that text messages could help to support empathy, the researchers also found that male participants who were trained to be more empathic were less likely to say that it was acceptable to behave aggressively. In addition, in a double-blind 6 month follow-up, participants who received the empathy-building messages were less likely to be verbally aggressive in response to a hostile text message sent from an unknown person (Konrath, et al., 2014).

Another study examined whether cognitive behavior therapy-based messages could help to reduce aggressive traits in high risk boys in Tehran (Rajabi, et al., 2012). The researchers randomly assigned 60 young adolescents from a behavior disorders center to receive 2 text messages a day for a 2 month period, or to a control condition (not described in paper). Sample messages included, "I can leave a place when I get angry" and "Others' behavior can have positive reasons." Participants in the treatment group declined in self-rated aggressive traits, while there were no changes in the control group. However, without knowing more about what exactly the control group experienced, and the procedures for randomization, it is not possible to make strong conclusions about this study.

Parenting. It is commonly observed that children do not come with their own instruction manual, and parenting can be stressful and difficult, especially for new parents in the first few years of their child's life. Although there are many apps available that claim to support parents in their childrearing, very few mobile technologies are evidence based. One program that was developed with rigorous scientific standards is the Estrellita app, which helps parents to record data and milestones related to their premature infant's development (Hirano, Tang, Cheng, \& Hayes, 2012). Although this app was rigorously developed, to date there are no published efficacy data.

Another rigorously developed program, text $4 b a b y$, sends health-related messages to expectant mothers in the hopes that these messages will increase positive health behaviors (e.g. getting immunizations) among mothers (Evans, Wallace, \& Snider, 2012). One recent pilot study randomly assigned 123 high-risk pregnant women to receive typical prenatal care only or the text4baby messages in additional to prenatal care for the duration of their pregnancy. Participants 
in the text4baby condition were almost 3 times more likely to report that they felt more ready to be a mother compared to those who only received traditional prenatal care. However, all other attitudes and behaviors were similar across the two groups (e.g. taking prenatal vitamins, avoiding alcohol, eating healthy foods). Thus, text messages during pregnancy can help pregnant women feel more confident about their readiness to be a parent. Even if this doesn't specifically change other outcomes, it is possible that this could translate into better parenting behaviors and child outcomes, however, more research is needed to test these hypotheses.

One small pilot study compared the efficacy of an evidence based parenting skills program for disruptive children (Jones et al., 2013). Participants were 15 low income parents of disruptive children who received either a traditional clinic-based program, Helping the Noncompliant Child (HNC), or a smartphone-enhanced version of the program (TE-HNC). HNC has two parts. The first part focuses on establishing positive social interactions and the second teaches parents how to give clear instructions and follow through on them. The smartphone enhancements included skills videos, daily surveys, phone and video calls from clinicians, video feedback of skills practice, and text message reminders. The study found that parents in the smartphone-enhanced (TE-HNC) group were more likely to attend weekly sessions and midweek calls, and were also more likely to complete the home practice requirements compared to the traditional treatment format (HNC). In addition, child behaviors improved more in the smartphone enhanced group compared to the traditional group. However, the small sample size and the fact that there were multiple treatments present in the TE-HNC group make it impossible to determine what exactly caused the results.

In the best available parenting-related study to date, 371 low income mothers of preschoolers who were at high risk for maltreatment participated in a randomized trial of a mobile-enhanced parenting skill intervention (Carta, Lefever, Bigelow, Borkowski, \& Warren, 2013). Participants were randomly assigned to one of three conditions: a Planned Activities Training (PAT) intervention, 2) the same intervention, plus cell phone support (CPAT), or 3) a waitlist control condition. Participants in the PAT and CPAT groups were taught to prevent difficult child behaviors via 10 strategies (e.g. plan activities in advance, give choices, ignore minor misbehavior). Then those in the CPAT group were sent daily individualized text messages, which included a reminder to use specific PAT strategies, queries about the recent use of them, suggestions for free community activities, and supportive messages. They also received a check in phone call once a week. At the 6 month follow up, participants in the CPAT condition used the most PAT strategies, had more objectively observed sensitivity and responsiveness to their children, and had fewer depressive symptoms and lower parental stress. In addition, the children in the CPAT condition had more positive behaviors. However, it is unclear which part of CPAT led to these positive outcomes: the daily text messages or the weekly phone calls. Future studies would need to examine the specific effect of the text messages versus the phone calls in supplementing the parent training.

Summary. Six studies (one with two outcomes) used mobile interventions to improve social relationships. Interestingly, nearly all of them exclusively use text messages and every single one of them used a randomized control trial with a control group. Still, the small sample sizes in most of these studies mean that this domain of mobile psychosocial interventions is also still in the pilot testing stage. Future research should continue to explore the potential to use mobile technology to build stronger relationship skills.

$<$ INSERT TABLE 2 HERE $>$

\section{PRACTICAL TIPS FOR GETTING STARTED}

There have been enough mobile-based physical health interventions that there are published guidelines on how to get started (Bradbury, Watts, Arden-Close, Yardley, \& Lewith, 2013; Heron \& Smyth, 2010). Below I combine these techniques into a single step-by-step guide. 


\section{A) Pre-development phase}

The first step is to identify the target population, and key behaviors for the intervention to target. The target population can help in identifying key behaviors, and/or researchers can use taxonomies of behavior change (C. Abraham \& Michie, 2008; Michie et al., 2013; Michie, van Stralen, \& West, 2011). Next, researchers should select the mode of delivery that they prefer (i.e. smartphone or text message), keeping in mind the tradeoffs of using one or the other. Researchers should then decide on whether they want quantitative or qualitative outcomes. Often both work well together, because quantitative outcomes are easier to collect, but qualitative outcomes allow researchers to better understand participants' responses. The next step is to identify an appropriate theory to guide the intervention. Research has found that theory-informed interventions have larger effect sizes (Webb, Joseph, Yardley, \& Michie, 2010).

\section{B) Develop intervention plan or experimental design}

The next phase involves finalizing the practical details of implementation. For example, researchers should decide when to introduce each part of the intervention, how to distribute these parts (e.g. random order), how to assess its efficacy, and so on. They must also decide on the frequency (e.g. once per day) and the duration of the intervention (e.g. two months).

There are a number of specific intervention strategies that can be used, and these are listed below (Klasnja \& Pratt, 2012):

1) Tracking information

a. Journaling, recording, charting data (e.g. moods).

b. Letting participants see their data and usage trends over time.

c. Automatic sensing of information (e.g. via mobile phone accelerometer).

2) Sending information

a. Direct content (e.g. information, tips, advice, instructions).

b. Reminders (e.g. to take a deep breath, to take medication, etc.).

3) Involving healthcare providers (in the case of physical or mental health)

a. Remote coaching (e.g. clinicians review patients' data and give feedback).

b. Symptom monitoring (e.g. indicators of poor health are flagged and sent to clinicians).

c. Automated feedback (e.g. "everything looks okay" or "you should call your doctor").

4) Leveraging social influence

a. Adding involvement of participants' close others may make interventions more effective. This could be via social support, competition, or modeling positive behaviors. However, such interventions are very rare.

5) Entertainment-based programs

a. As an add on to the direct content portion (e.g. giving news or weather information in addition to tips about quitting smoking).

b. As the main intervention (e.g. see gamesforhealth.org).

An appropriate control group is needed for randomized control trials. What will this group receive? Is it fully matched to the treatment group, only differing on the key construct of interest? How will random assignment be determined and implemented so that research team members are blind to participants' condition? (Typically in mobile-based interventions, it is difficult for participants to remain blind to condition.) It's important to imagine the study from the users' perspective. From the consent to the debriefing process, what concerns might participants have (e.g. privacy, confidentiality, too much work, etc.)? How can they be alleviated? It is also important to consider the research team's time, energy, expertise, and available funds, and to cut unnecessary pieces of the intervention. A power analysis should be run to determine an adequate sample size. It is easy to get excited about the potential intervention and try to do too much, especially at the pilot phase. Researchers should think in terms of "must have" versus "should have" versus "could have" versus "would like" features of the intervention and the study. 
There are a number of ways to involve participants in the intervention. For example, participants can choose their preferred frequency and timing of the intervention, or this can be determined by the researcher. In SMS studies, messages can be unidirectional (sent from researcher only) or they can involve some 2-way communication. If the messages are interactive, researchers should determine which components are interactive, and whether there will be automatic feedback or a real person responding to the messages. Some research tailors the interventions to individuals, which may increase their efficacy (Fjeldsoe, et al., 2009). Examples of tailoring include using participant's name or demographics (gender, age), having participants write their own messages, and tailoring to their personal goals.

\section{C) User testing}

The goal of the user testing phase is to identify problems with the study, barriers to participation, different potential drop out points, and the optimal frequency and duration of the intervention. Researchers should make changes based on user feedback, and then test the revised intervention on other users until there are few changes to be made.

\section{D) Feasibility or pilot study}

For the initial pilot study, it is best to use objective and validated outcome measures (Fjeldsoe, et al., 2009; Heron \& Smyth, 2010). Pilot tests can then be refined into larger randomized trials. As can be seen from Table 2, many studies have small sample sizes, making it difficult to detect statistically significant effects (Fjeldsoe, et al., 2009).

\section{FUTURE DIRECTIONS}

To date, there are only 23 known studies targeting psychosocial outcomes, and of those, only 4 consist of a randomized control trial with a large sample and an unconfounded treatment. Clearly, much work needs to be done to better understand the conditions under which mobilebased interventions can effectively address psychosocial issues.

\section{Quality of research studies and interventions}

The vast majority of mental health apps lack scientific evidence for their efficacy (Donker, et al., 2013; Gaggioli \& Riva, 2013; Mohr, Burns, Schueller, Clarke, \& Klinkman, 2013). "Industry has begun developing and marketing behavior intervention technologies for a wide variety of mental health problems. These are frequently brought to market with little or no evaluation of their efficacy or utility" (Mohr, et al., 2013), p. 333). Thus, more randomized control trials are needed. In addition, the majority of studies to date have extremely small sample sizes, which limits their ability to find significant effects. Moreover, there is a need for more long-term follow ups after the post-intervention period. Such follow ups are extremely rare (see Table 1), but important in order to determine whether effects last beyond the treatment period (Heron \& Smyth, 2010). Finally, researchers need to report the results of ecological momentary assessments, most of which are not reported because of complex statistical requirements (Heron \& Smyth, 2010).

The quality of the interventions themselves also needs improvement. There are currently no clear standards for developing and implementing these technological interventions, which is especially problematic when working with clinical populations. Interventions need to be manualized and standardized so that they can be more widely disseminated at a high level of quality (Heron \& Smyth, 2010). Within the domain of mobile-based psychosocial interventions, this chapter is the only known review. There is a need for more reviews, and especially metaanalyses, in order to determine the most effective features of interventions (e.g. whether there are 2-way communication features or not, whether there is any tailoring). With respect to tailoring, for example, some research suggests that tailored interventions are more effective (Park, et al., 2014), whereas others find no clear advantages for tailored interventions (Heron \& Smyth, 2010). Mobile technology allows for a more personalized tailoring, one that is not based on broad demographic groups (e.g. gender, age) or shallow characteristics (e.g. one's name), but one that is 
based on one's own unique pattern of responding. For example, responsive tailoring based on users' own physiological or mood changes would likely have a higher impact than general tailoring. In any case, studies need to systematically vary the type and timing of tailoring to maximize efficacy.

Dosage levels should also be varied to determine the optimal frequency and duration of intervention messages. If we truly want to take seriously the idea that mobile interventions can help promote mental health and well-being, then we should treat dosage with the same care as pharmaceutical trials do with medications. On that vein, studies should attempt to calculate the cost effectiveness of interventions, both for participants (or patients) and for researchers (or clinicians). How do mobile technology interventions compare to other available treatments in terms of costing money and time? Studies rarely report such information.

There is also a need for comparisons between SMS versus smartphone app interventions, and mobile-alone versus mobile-plus (e.g. paired with therapy) interventions. It is unclear if they are equally efficacious (Heron \& Smyth, 2010). In addition, there is virtually no research on mechanisms. Most research simply reports that the intervention caused the outcome, without determining why. Ecological momentary assessment data can help with this. For example, perhaps a cognitive behavior therapy intervention delivered by text message is effective for reducing depressive symptoms because it makes participants more likely to socialize with others.

\section{Generalizability of research}

There needs to be more representative and diverse samples in mobile intervention research (Fjeldsoe et al, 2009), including more diverse cross-cultural samples. People in developing countries have low access to mental health services. Yet there is a relatively high (and growing) penetration of mobile technology in these countries (Aggarwal, 2012; Farrington, Aristidou, \& Ruggeri, 2014; Torous, 2013). To date, most mobile-based experiments have been conducted in North America or Europe. There is great potential to use this technology in developing countries, since the programs need not be expensive or technologically sophisticated to be effective (Farrington, et al., 2014). However, researchers and practitioners also must consider potential problems (e.g. adaptability to new groups, less education regarding mental health, stigmatization of mental health problems, comorbidities of mental health conditions). There is a need for flexible intervention systems that are "capable of addressing a range of disorders through a range of mobile capabilities and in a range of contexts" (Farrington et al, 2014, p. 4).

There is also a need to generalize to other psychosocial topics. For example, no known studies have used this type of technology to implement more theoretically-focused social science experiments. Much could be learned by using these technologies to better understand general psychological principles. For example, researchers could better understand how cultural beliefs develop and change over time based on changes in context (Heine \& Lehman, 2004; Oyserman \& Lee, 2007), or the extent to which certain contexts affect what are typically seen as fixed cognitive skills (Houben, Nederkoorn, Wiers, \& Jansen, 2011; Orr \& Weissman, 2011).

\section{Technological roadblocks}

Most academic researchers do not have programming experience and do not want to invest time into learning complex computer programs in order to implement mobile-based interventions. Typically they partner with programmers or technology companies in order to implement their desired interventions. This greatly limits the number of researchers who are willing and able to use mobile-based tools. In the early days of internet research, there were similar technological roadblocks. The development of user-friendly internet research portals (e.g. Survey Monkey, Qualtrics) led to a large increase in the number of internet-based studies. Only a few such tools exist to date (see Table 3). The most promising of those is called SurveySignal (Hofmann \& Patel, 2014). It allows researchers to log onto an easy-to-navigate web portal and specify the frequency and duration of survey messages ("signals") to be sent to participants, for a low price (10 cents per signal). Currently it is limited to collecting survey data via ecological 
momentary assessments, but the company is prototyping ecological momentary interventions that could be sent by text message or email. The development of researcher-friendly tools to assist with study implementation and data collection is a necessary next step in the field of mobilebased interventions.

\section{$<$ INSERT TABLE 3 HERE $>$}

\section{Conclusion}

Although research on mobile interventions is more established in physical health domains, research on psychosocial outcomes is only emerging. So far, the majority of studies have focused on mental health outcomes, with very few studies moving beyond specific mental health conditions and into psychological wellbeing and strengthening social relationships. Moreover, no known studies have used this type of technology to understand more basic research questions (i.e. those that are designed to increase our understanding of a construct) rather than more applied research questions (i.e. those that are designed to address a specific problem in the real world). In addition, there are very few high quality randomized control trials, and virtually no research in developing nations. Thus, the full potential of mobile technologies in psychosocial domains is yet to be discovered. 